\title{
Observation of a Space-Time Crystal in a Superfluid Quantum Gas
}

\author{
J. Smits, ${ }^{1}$ L. Liao, ${ }^{2}$ H. T. C. Stoof,${ }^{2}$ and P. van der Straten ${ }^{1, *}$ \\ ${ }^{1}$ Debye Institute for Nanomaterials Science and Center for Extreme Matter and Emergent Phenomena, Utrecht University, \\ P.O. Box 80.000, 3508 TA Utrecht, Netherlands \\ ${ }^{2}$ Institute for Theoretical Physics and Center for Extreme Matter and Emergent Phenomena, Utrecht University, \\ P.O. Box 80.000, 3508 TA Utrecht, Netherlands
}

(Received 16 July 2018; published 29 October 2018)

\begin{abstract}
Time crystals are a phase of matter, for which the discrete time symmetry of the driving Hamiltonian is spontaneously broken. The breaking of discrete time symmetry has been observed in several experiments in driven spin systems. Here, we show the observation of a space-time crystal using ultracold atoms, where the periodic structure in both space and time is directly visible in the experimental images. The underlying physics in our superfluid can be described $a b$ initio and allows for a clear identification of the mechanism that causes the spontaneous symmetry breaking. Our results pave the way for the usage of space-time crystals for the discovery of novel nonequilibrium phases of matter.
\end{abstract}

DOI: 10.1103/PhysRevLett.121.185301

Wilczek proposed the idea of time crystals in 2012 [1], where in analogy to space crystals continuous time symmetry is broken spontaneously. Since that time there has been discussion on what should constitute a time crystal [2,3] and how to create them. Watanabe and Oshikawa [4] showed that, in principle, continuous time symmetry cannot be broken spontaneously into discrete symmetry in the ground state. However, there have been proposals to realize instead a discrete time crystal by breaking of discrete time translation symmetry [5-9]. Following a theoretical model by Yao et al. [10], several experiments [11-14] realized this particular symmetry breaking in driven spin systems. These experiments were limited to probing a very restricted number of particles [11] or an ensemble of particles without any spatial resolution [12-14], preventing the direct observation of spatial ordering.

In this Letter, we report the direct observation of a spacetime crystal exhibiting not only periodic oscillations in time with double the period of the driving force, but also an oscillatory spatial structure; i.e., both discrete time translation symmetry as well as continuous spatial translation symmetry are broken. Because of the small dissipation in our superfluid gas, we can study the space-time crystal over an extensive period of time, showing the collapse and revival of the oscillating long-lived spatially ordered state. Superfluid quantum gases are the ideal system to study discrete time-crystals. Because of the low viscosity and heat conduction, excitations in the system can be induced without the associated heating of the system. Periodic driving of the excitations in the system can easily be arranged due to the harmonic confinement of the atoms in the trap. Crucial in the driven spin systems $[11,12,15]$ has been the occurrence of strong disorder, where either many-body localization or some other mechanism is the cause for the small dissipation in the experiments.

However, as shown by Else, Bauer, and Nayak [16], time crystals can also exist in the prethermal regime, if the drive frequency is sufficiently large compared to the excitation frequency. Following these experiments, there have been a large number of proposals [17-22] for the observation of time crystals using several different systems (see also the review in Ref. [23]). In superfluid quantum gases, disorder is absent. Since superfluid quantum gases can be imaged using phase-contrast techniques, which allows the accumulation of several tens of images of the same superfluid cloud, the dynamics of the system can be studied over many cycles. Moreover, as the conditions of the space-time crystal are not very sensitive to the initial drive of the excitations, the superfluid cloud can be studied over a prolonged period of time by combining multiple measurement series together, extending the observation period to several seconds. Finally, the dynamics of the superfluid quantum gas in a radial symmetric trap can be simulated using time-splitting spectral methods [24], which allows us to compare our experimental findings with simulations to elucidate the mechanisms behind the space-time crystal formation.

The superfluid is produced in the trap in a cigar-shaped form, where the ratio between the trap frequencies causes the axial size to be about 40 times larger than the radial size. After sample preparation [25], the radial trap frequency is suddenly perturbed, and this induces a radial breathing mode of the cloud with a frequency of $f_{D}=$ $104.691(16) \mathrm{Hz}$, which is only weakly damped and has a decay time of several seconds. This radial breathing mode with a period $T_{D}=1 / f_{D}$ acts as the drive for the excitation of the cloud in the axial direction. After many radial oscillations, a high-order excitation emerges in the axial 


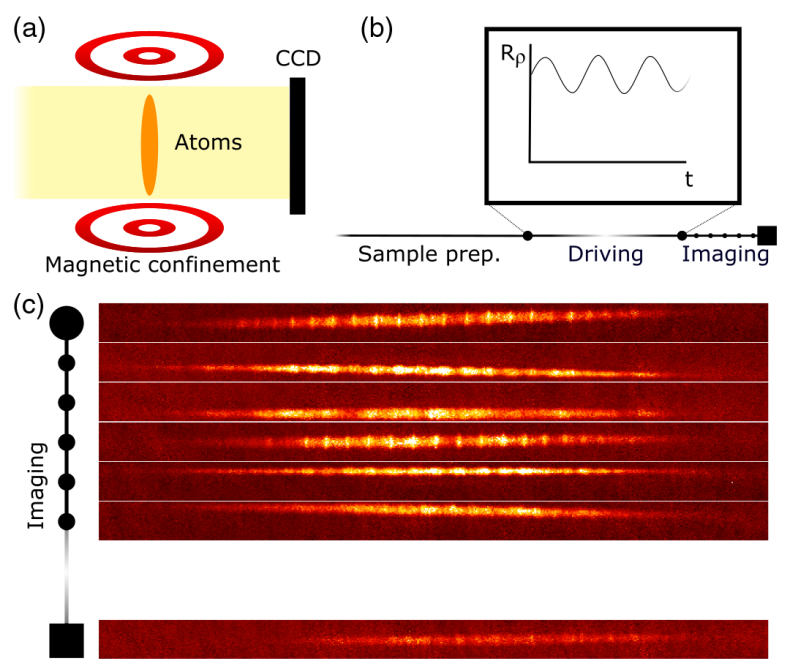

FIG. 1. Schematic representation of the experimental setup, the timeline, and the imaging sequence. (a) Schematic view of the imaging system and atomic cloud. (b) Experimental sequence and schematic representation of the driving. (c) Imaging sequence showing the first six and the last image of a selected run. In total, 50 images are taken each run with $3.28 \mathrm{~ms}$ between images.

direction, which has been observed previously and interpreted in that paper as "Faraday waves" [35]. By observing the spatiotemporal long-range order, we show that an interpretation as a space-time crystal is more appropriate using the modern language of nonequilibrium phase transitions. Figure 1 shows several images of the pattern displaying the large variety in radial size and axial excitation. This axial pattern is observed only if the radial breathing mode is strongly excited and the perturbation of the cloud is in the nonlinear regime.

To study the axial pattern, the density profile is integrated over the radial direction, and the result is shown in Fig. 2 as a function of the time. A lattice of maxima in the

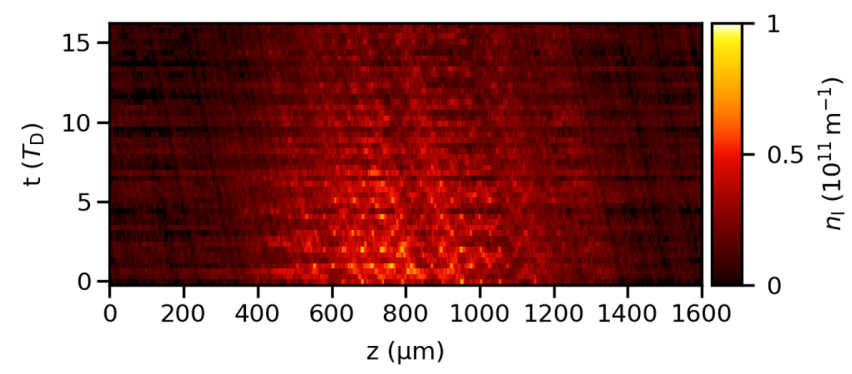

FIG. 2. Line density $n_{\ell}$ as a function of the time and position starting $500 \mathrm{~ms}$ after the onset of the drive. Time slices are taken from a single experimental run. In both space and time, a recurring pattern is observed. The (temporal) period of the pattern corresponds to twice the breathing period $T_{D}$. The diagonal streaks in the image are caused by a correction for the uncoupled axial center-of-mass motion and darker areas in the imaging. The decrease of the signal is attributed to a slight particle loss (3\% per shot) due to interactions of imaging light with the atoms. density is observed in both the temporal and spatial directions, a clear signature of a space-time crystal. The wave number of the pattern increases slightly towards the edges of the superfluid, which is attributed to the finite extent of the cloud. The period of the pattern is determined to be almost $2 T_{D}$ over the entire detection period, and this subharmonic response to the drive is a requirement for the symmetry breaking implied by a discrete time crystal.

In Figs. 3(a) and 3(c), the central part of the axial profile of Fig. 2 is shown just after the start of the drive [Fig. 3(a)] and after the axial excitation pattern emerged [Fig. 3(c)]. Figure 3(c) shows that the space-time crystal has a centered cubic lattice structure with a period $2 T_{D}$ in time. To determine the long-range temporal and spatial order, these patterns are Fourier transformed and shown in Figs. 3(b) and 3(d), respectively. The Fourier signal for the axial excitation pattern in Fig. 3(d) contains four Fourier peaks at $\left(k / k_{c}, f / f_{D}\right)=( \pm 1, \pm 1 / 2)$, where the temporal frequency is half the driving frequency $f_{D}=1 / T_{D}$. This again shows that we are dealing with a discrete time crystal. The spatial periodicity $2 \pi / k_{c}$ is $57.3 \mu \mathrm{m}$ as determined from the axial mode that we excite [25]. The appearance of the narrow peaks in the (momentum-frequency) Fourier plane is a clear indication of the simultaneous spatial and temporal long-range order in our system and manifestly indicates that we can truly speak of a space-time crystal. The Fourier signals in Figs. 3(b) and 3(d) also contain two peaks in the temporal signal for nonzero frequencies at $f \simeq \pm f_{D}$, indicating the excitation of a weakly excited scissor mode. Such a mode can easily be induced due to small imperfections in the fabrication of the magnetic trap.

In order to further check the validity of our experimental findings, we have numerically simulated the evolution of a Bose-Einstein condensation using a time-splitting spectral method under the same conditions regarding the number of atoms, the trap frequencies, and the drive assuming a radial-symmetric trap [25]. The results are shown in Figs. 3(e)-3(h) and show excellent agreement with the experimental results apart from the weak scissor mode, which is absent in the simulations. This agreement shows that the physics of the space-time crystal for our experimental conditions is fully encapsulated in the GrossPitaevskii equation.

To demonstrate the longevity of the space-time crystal, we compare the amplitude of the driving mode to the crystal fraction. The amplitude of the drive and emergence of the crystalline phase are shown in Fig. 4. Over a full experimental run of $2.6 \mathrm{~s}$, the pattern is seen to appear and disappear two times. The appearances of the space-time crystal occur at times $t=350$ and $1350 \mathrm{~ms}$, while the disappearance of the crystalline phase coincides with the decrease of the driving mode amplitude to near zero. The space-time crystal lasts, in each individual appearance, for over $500 \mathrm{~ms}$ or $50 T_{D}$. The decrease of the driving mode is caused by the coupling to the scissor mode. 

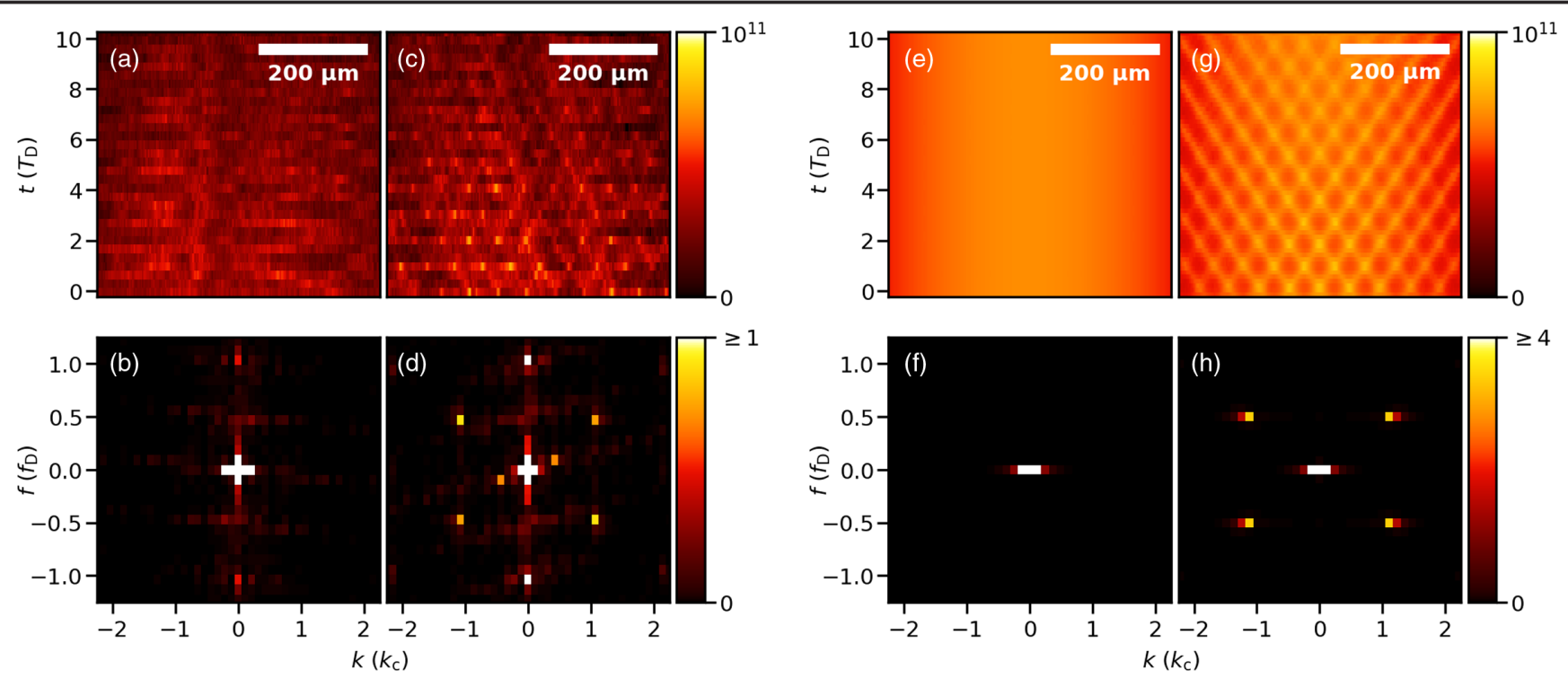

FIG. 3. Fourier analysis and comparison of the experiment with the simulation. (a) Line density at the center of the cloud before the onset of the space-time crystalline phase, directly after the excitation. (b) Fourier transform of the data in (a). Peaks at $f / f_{D}= \pm 1$ are associated with a weakly excited scissor mode. The signal around the origin is associated with the equilibrium profile of the condensate. (c) Take-out of Fig. 2. Line density at the center of the cloud after the transition to the space-time crystalline phase, after a driving time of $500 \mathrm{~ms}$. A lattice has formed. (d) Fourier transform of the data in (c), with the appearance of four additional peaks due to the space-time crystal at $\left(k / k_{c}, f / f_{D}\right)=( \pm 1, \pm 0.5)$, where $k_{c}$ is the center wavelength [25]. (e) Simulated line density for a modulation depth of 0.02 , after a wait time of $25 T_{D}$. (f) Fourier transform of the data in (e). Notice that in the simulation only the equilibrium profile is visible. (g) Simulated line density for a modulation depth of 0.2 after a wait time of $25 T_{D}$. A pattern similar to the experimental data of (c) is observed. (h) Fourier transform of ( $\mathrm{g}$ ). Note the appearance of the four additional peaks at $\left(k / k_{c}, f / f_{D}\right)=( \pm 1, \pm 0.5)$ attributed to the space-time crystal. The line density in (a), (c), (e), and (g) is in units of $10^{11}$ atoms $/ \mathrm{m}$. Fourier images in (b), (d), (f), and (h) are truncated and normalized to 1 for the experimental data.

The periodicity in the occurrence of the space-time crystal coincides approximately with the period that we extract from our simplified model describing the coupling between scissor and breathing mode [25]. The scissor mode has a period of about $T_{D} / 2$ and is not linearly coupled to the axial excitation pattern due to parity conservation.
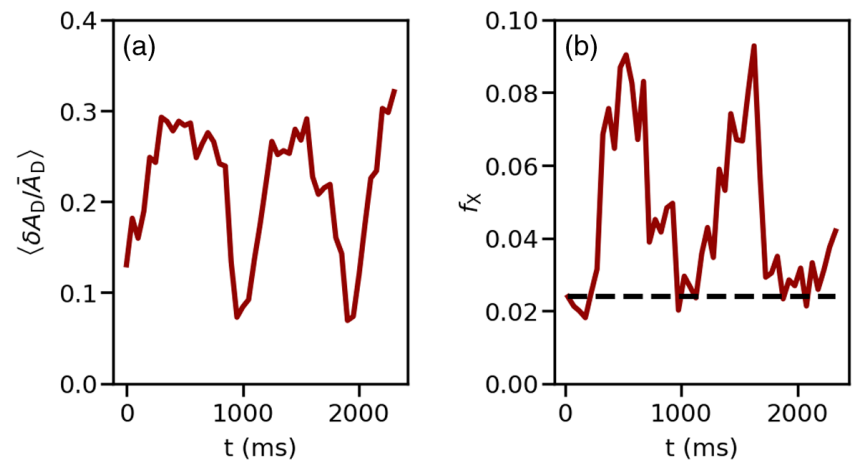

FIG. 4. Long-term behavior of the amplitudes of the drive and crystal fraction. (a) Relative amplitude of the radial breathing mode derived by fitting a two-dimensional profile to the data. (b) Crystal fraction determined from each measurement run. The dashed line indicates the background signal from shot-to-shot noise. Notice that the crystalline phase appears a certain time after the driving mode revives [25].
Theoretically, we treat the space-time crystal variationally as a multimode system with the mode functions $P_{4 j+2}(\tilde{z})-P_{4 j}(\tilde{z})$, with $\tilde{z}=z / R_{z}(t)$ in terms of Legendre polynomials, and frequencies $f_{j}$ excited by the drive due to the time dependence of the Thomas-Fermi radii $R_{x}(t)$, $R_{y}(t)$, and $R_{z}(t)$, for which $R_{i}(t)=R_{i}\left(t+T_{D}\right)$ and $i=x$, $y$, and $z$. After substituting this ansatz in the action for the Gross-Pitaevskii equation and neglecting nonlinear mode coupling, we ultimately obtain the Hamiltonian

$\hat{H}=\sum_{j}\left[2 \pi \hbar f_{j} a_{j}^{\dagger} a_{j}+g_{j}(t)\left(a_{j}^{\dagger} a_{j}^{\dagger}+a_{j} a_{j}+2 a_{j}^{\dagger} a_{j}\right)\right]$,

where $a_{j}^{(\dagger)}$ are the annihilation (creation) operators for quanta in the mode $j$ and $g(t)$ is the coupling with the periodicity of the drive. By moving to the rotating frame and applying the rotating-wave approximation to eliminate the time dependence of the drive $g(t)$, we find the effective Hamiltonian

$\hat{H}_{\text {eff }}=\sum_{j}\left[2 \pi \hbar\left(f_{j}-f_{D} / 2\right) a_{j}^{\dagger} a_{j}+g_{j, 0}\left(a_{j}^{\dagger} a_{j}^{\dagger}+a_{j} a_{j}\right)\right]$,

where $g_{j, 0}$ is proportional to the amplitude of the drive. Note that this yields a Hamiltonian which is time independent in 


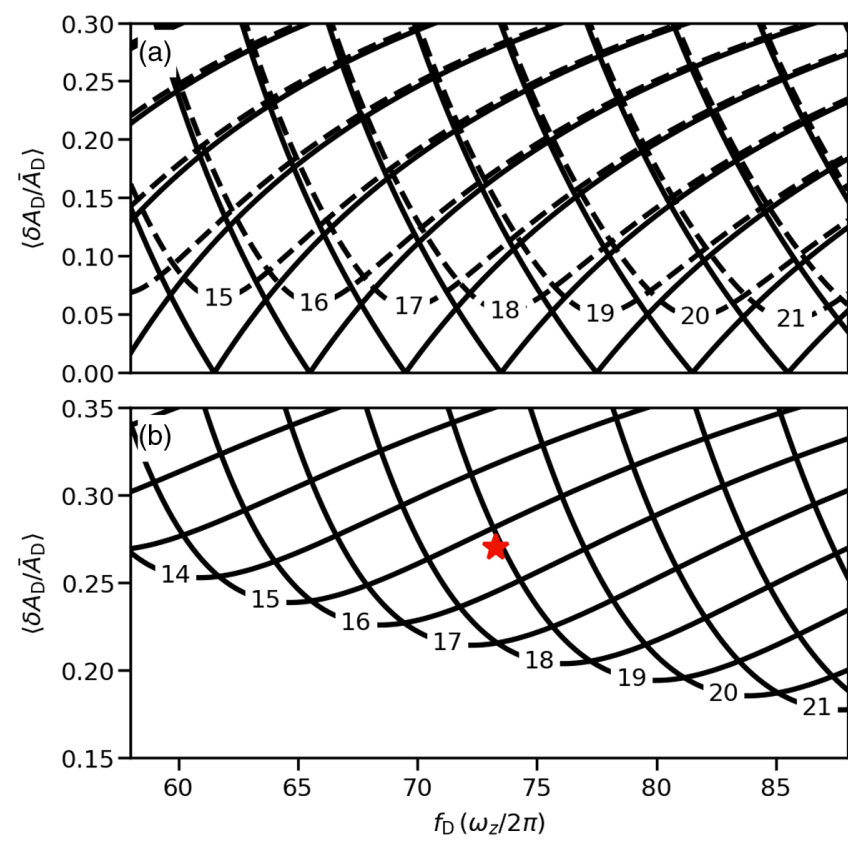

FIG. 5. Minimum required driving amplitude $\left\langle\delta A_{D} / \bar{A}_{D}\right\rangle$ as a function of the driving frequency $f_{D}$ in a linear response analysis [36]. (a) Without damping (solid line) a mode $j$ can be driven with an arbitrary small amplitude, if the driving frequency coincides with twice the mode frequency $f_{j}$, whereas for small damping (dashed line) there is for any drive frequency a threshold, below which the mode is not excited. (b) Under our experimental conditions (indicated by the red star), the damping is larger and the threshold for exciting modes becomes larger. In the case of linear response, the modes $j=16,17$, and 18 can be excited, and, depending on the competition between these modes, one of them dominates the pattern [36].

the rotating frame and that represents the appropriate Hamiltonian for prethermalization of the system.

The mode that is observed depends on the driving frequency $f_{D}$ and the driving amplitude $\left\langle\delta A_{D} / \bar{A}_{D}\right\rangle$ [36]. In Fig. 5, the minimum required amplitude is shown as a function of the driving frequency. In the absence of damping, as shown in Fig. 5(a), a mode $j$ can be driven with an arbitrary small amplitude, if the resonance condition $2 f_{j}=f_{D}$ is fulfilled. In the case of damping, the threshold for exciting the pattern becomes finite. Applying the analysis of Ref. [36] to our experimental conditions [see Fig. 5(b)] shows that the driving amplitude used in our experiment is sufficient to excite several modes $j$ and the competition between these modes causes one of the modes to grow exponentially and thus dominate the observed pattern.

The Hamiltonian of Eq. (2) explicitly breaks the $U(1)$ symmetry $a \rightarrow a e^{i \vartheta}$. This implies that in the laboratory frame $\left\langle a_{j} a_{j}\right\rangle \propto e^{-2 \pi i f_{D} t}$ is always nonzero and oscillates with the period of the drive. However, there is an additional $\mathbb{Z}_{2}$ symmetry $a_{j} \rightarrow-a_{j}$, which is spontaneously broken when $\left\langle a_{j}\right\rangle \neq 0$, which occurs when the mode is Bose condensed. This leads to the appearance of the time dependence $\left\langle a_{j}\right\rangle \propto e^{-\pi i f_{D} t}$ in the laboratory frame. The breaking of this $\mathbb{Z}_{2}$ symmetry thus leads to an oscillation with period $2 T_{D}$. We propose that for low occupation $\left(\left\langle a_{j}\right\rangle \simeq 0\right)$ the system is in a state dominated by a description based on the evolution of the pair correlation $\left\langle a_{j} a_{j}\right\rangle$. As occupation in the mode grows, i.e., the occupation number of the mode $\left\langle a_{j}\right\rangle$ goes up, there is a phase transition from the paired state to a state dominated by dynamics in $\left\langle a_{j}\right\rangle$, breaking the $\mathbb{Z}_{2}$ symmetry. We identify this transition as the phase transition to the time crystal.

In summary, we have shown the existence of a spacetime crystal which is robust against fluctuations in experimental parameters and long-lived. Future experiments are aimed at studying elementary excitations such as solitons and sound in the presence of a space-time crystal, as our system is an excellent testing ground for these excitations. Moreover, it can be explored whether this spatially ordered state has supersolid properties, as this would allow the study of out-of-equilibrium supersolids [37,38], combining the fields of time crystals and supersolids and exploring a currently unknown corner of physics.

We thank Alexander Groot and Pieter Bons for their contribution to the initial stages of this research. This work is supported by the China Scholarship Council (CSC) and the Stichting voor Fundamenteel Onderzoek der Materie (FOM), which is part of the Netherlands Organization for Scientific Research (NWO), and is part of the D-ITP consortium, a program of the NWO that is funded by the Dutch Ministry of Education, Culture and Science (OCW).

*p.vanderstraten@uu.nl

[1] F. Wilczek, Phys. Rev. Lett. 109, 160401 (2012).

[2] P. Bruno, Phys. Rev. Lett. 110, 118901 (2013).

[3] P. Nozières, Europhys. Lett. 103, 57008 (2013).

[4] H. Watanabe and M. Oshikawa, Phys. Rev. Lett. 114, 251603 (2015).

[5] D. V. Else, B. Bauer, and C. Nayak, Phys. Rev. Lett. 117, 090402 (2016).

[6] K. Sacha, Phys. Rev. A 91, 033617 (2015).

[7] L. Guo, M. Marthaler, and G. Schön, Phys. Rev. Lett. 111, 205303 (2013).

[8] V. Khemani, A. Lazarides, R. Moessner, and S. L. Sondhi, Phys. Rev. Lett. 116, 250401 (2016).

[9] C. W. von Keyserlingk, V. Khemani, and S. L. Sondhi, Phys. Rev. B 94, 085112 (2016).

[10] N. Y. Yao, A. C. Potter, I.-D. Potirniche, and A. Vishwanath, Phys. Rev. Lett. 118, 030401 (2017).

[11] J. Zhang et al., Nature (London) 543, 217 (2017).

[12] S. Choi et al., Nature (London) 543, 221 (2017).

[13] J. Rovny, R. L. Blum, and S. E. Barrett, Phys. Rev. Lett. 120, 180603 (2018).

[14] S. Pal, N. Nishad, T. S. Mahesh, and G. J. Sreejith, Phys. Rev. Lett. 120, 180602 (2018). 
[15] J. Rovny, R. L. Blum, and S. E. Barrett, Phys. Rev. B 97, 184301 (2018).

[16] D. V. Else, B. Bauer, and C. Nayak, Phys. Rev. X 7, 011026 (2017).

[17] B. Huang, Y.-H. Wu, and W. V. Liu, Phys. Rev. Lett. 120, 110603 (2018).

[18] K. Sacha, Phys. Rev. A 91, 033617 (2015).

[19] E. Lustig, Y. Sharabi, and M. Segev, arXiv:1803.08731.

[20] W. W. Ho, S. Choi, M. D. Lukin, and D. A. Abanin, Phys. Rev. Lett. 119, 010602 (2017).

[21] K. Mizuta, K. Takasan, M. Nakagawa, and N. Kawakami, Phys. Rev. Lett. 121, 093001 (2018).

[22] A. Russomanno, F. Iemini, M. Dalmonte, and R. Fazio, Phys. Rev. B 95, 214307 (2017).

[23] K. Sacha and J. Zakrzewski, Rep. Prog. Phys. 81, 016401 (2018).

[24] W. Bao, D. Jaksch, and P. A. Markowich, J. Comput. Phys. 187, 318 (2003).

[25] See Supplemental Material at http://link.aps.org/ supplemental/10.1103/PhysRevLett.121.185301 for experimental conditions, methods for data analysis, and a short description of our theoretical model, which includes Refs. [24,26-34].

[26] R. Meppelink, R. A. Rozendaal, S. B. Koller, J. M. Vogels, and P. van der Straten, Phys. Rev. A 81, 053632 (2010).
[27] C. J. Pethick and H. Smith, Bose-Einstein Condensation in Dilute Gases, 2nd ed. (Cambridge University Press, Cambridge, England, 2008).

[28] E. Zaremba, Phys. Rev. A 57, 518 (1998).

[29] W. Kohn, Phys. Rev. 123, 1242 (1961).

[30] D. Guéry-Odelin, F. Zambelli, J. Dalibard, and S. Stringari, Phys. Rev. A 60, 4851 (1999).

[31] F. Chevy, V. Bretin, P. Rosenbusch, K. W. Madison, and J. Dalibard, Phys. Rev. Lett. 88, 250402 (2002).

[32] O. M. Maragò, S. A. Hopkins, J. Arlt, E. Hodby, G. Hechenblaikner, and C. J. Foot, Phys. Rev. Lett. 84, 2056 (2000).

[33] U. Al Khawaja and H. T. C. Stoof, Phys. Rev. A 65, 013605 (2001).

[34] H. Stoof, K. Gubbels, and D. Dickerscheid, Ultracold Quantum Fields (Springer, New York, 2009).

[35] P. Engels, C. Atherton, and M. A. Hoefer, Phys. Rev. Lett. 98, 095301 (2007).

[36] L. Liao, J. Smits, P. van der Straten, and H. Stoof (to be published).

[37] J. Leonard, A. Morales, P. Zupancic, T. Esslinger, and T. Donner, Nature (London) 543, 87 (2017).

[38] J.-R. Li, J. Lee, W. Huang, S. Burchesky, B. Shteynas, F. a. Top, A. O. Jamison, and W. Ketterle, Nature (London) 543, 91 (2017). 